\title{
NUMERICAL EXPERIMENTS ON VORTEX SHEDDING FROM AN OSCILLATING CYLINDER
}

\author{
Fernando Ponta ${ }^{*}$, Hassan Aref ${ }^{* *}$ \\ *Department of Theoretical and Applied Mechanics, University of Illinois at Urbana-Champaign, \\ Urbana, IL 61801, USA \\ *** Virginia Polytechnic Institute \& State University, Blacksburg, Virginia, 24061-0217, USA
}

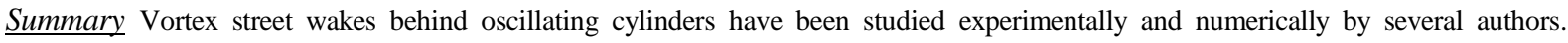
Williamson and Roshko attempted a classification of the various wake patterns observed as a function of two dimensionless parameters, the wavelength and amplitude of the undulatory motion of the cylinder scaled by the cylinder diameter. Several qualitatively distinct wake regimes were observed experimentally. These were classified in terms of the vortex patterns, e.g., two singlets, two pairs, pair and singlet, and so on. We have performed a number of numerical experiments for $\mathrm{Re}=140$ and established several points of correspondence with the experiments. Our simulation results also shed light on the classification scheme of Williamson and Roshko and suggest how this classification may change with Reynolds number. We find remarkable sensitivity to details of the oscillation of the cylinder, in particular whether the oscillation takes place at fixed streamwise velocity or at fixed cylinder speed along its trajectory.
\end{abstract}

\section{INTRODUCTION}

In addition to the familiar Kármán vortex street behind a stationary cylinder in a uniform stream several more complex vortex patterns have been observed in the wakes of oscillating cylinders. There is a large body of literature on experimental studies of vortex wakes behind cylinders oscillating either perpendicularly to or in-line with the uniform stream (see [2] for further references). An attempt at classifying such vortex patterns has been made by Williamson and Roshko [3], henceforth referred to as WR, using a symbolic code of letters and numbers that describes the combination of pairs and single vortices shed during each cycle of the forced oscillation of the cylinder. For example, $2 \mathrm{~S}$ indicates two single vortices shed per cycle, $2 \mathrm{P}$ indicates two vortex pairs shed per cycle, $\mathrm{P}+\mathrm{S}$ signifies a pattern in which one pair and a single vortex are shed in each cycle, and so on. WR gave a map of when the various vortex patterns would occur using a non-dimensional wavelength-amplitude plane. We have used their classification and map as a basis for our computational study. Besides the experimental work referenced above, there is some analytical and computational work on complex wake structures of forced and freely oscillating cylinders. Aref and Stremler [1] applied their analysis of the motion of three point vortices with net zero circulation in a periodic strip to the observations of WR of the P+S wake and were able to find reasonable correspondence with the experimental results although several parameters (e.g., the vortex circulations) could be adjusted. This analysis suggested that many additional possibilities, so far not observed experimentally, exist for the vortex structure of the wake behind an oscillating cylinder. We have begun referring to such vortex patterns as exotic wakes. Several direct numerical simulation studies have been performed on freely oscillating cylinders for Re up to 350 (see [2] for a list of references). In this paper we primarily study the periodic vortex structures produced in the wake of a cylinder subject to forced oscillations at low Re, exploring in particular the phenomenon of the $\mathrm{P}+\mathrm{S}$ structure. To this end, we have developed a new numerical approach that we briefly introduce in the next Section. Using this code, we experiment with wakes of oscillating cylinders, exploring a section of the WR map at $\mathrm{Re}=140$ and identifying similarities and differences with the higher Re results reported in WR.

\section{THE KINEMATIC LAPLACIAN EQUATION METHOD}

In this section we briefly introduce the main features of our method for computing the time-dependent flow in the wake of an oscillating cylinder. Our method can be seen as a vorticity creation algorithm or, alternatively, as a time-space split method to solve the time-dependent viscous flow around moving bodies. This new type of vorticity creation method is based on a space-time splitting of the problem that solves the time evolution of the vorticity as an ODE on each node of the spatial discretization, using at each step of the time discretization the spatial solution for the velocity field provided by a new PDE expression that we call the Kinematic Laplacian Equation (KLE). The idea is based on a kinematic relation that solves the Laplacian of the velocity field for a given distribution of vorticity and rate of expansion. The vorticity distribution is provided by the ODE solution and the rate of expansion is zero due to incompressibility. The KLE is intended to work within non-linear time-evolving schemes providing a quick, linear, spatial solution to the velocity field for successive time-steps. For time integration we used a variable-order Adams-Bashforth-Moulton predictor-corrector solver with adaptive stepsize control, which proved to be quite efficient for this application. For the discretization of the KLE we used unstructured meshes of isoparametric finite elements.

\section{NUMERICAL EXPERIMENTS ON VORTEX WAKES OF AN OSCILLATING CYLINDER}

In this section we report on our results regarding the formation, shedding and further evolution of vortex patterns produced in the wake of a cylinder executing forced oscillations for the range of Reynolds number below 180. This is, 
approximately, the periodic laminar wake regime for the usual, Kármán vortex street case. We concentrated on the case of $\mathrm{Re}=140$ since this case is representative of the range and shows a variety of arrangements of vortex structures. Figure 1(a) shows the non-dimensional vorticity field for wavelength $=7.5$ and amplitude $=1$ (both in units of the cylinder diameter), which produced the clearest $\mathrm{P}+\mathrm{S}$ arrangement. We checked that for each triplet the sum of the circulation of the two positive vortices equals the opposite of the circulation of the negative vortex, so the complete circulation produced in one cycle is very close to zero. We see how the positive (lower) half of the near wake is stretched by the strain rate field of the neighboring vortices until it breaks into two separated vortices. If we follow the evolution of the negative (upper) half of the near wake, we notice that it also starts to split in two but, contrary to what happens to its positive counterpart, a major portion of the shed vorticity remains together, and this vorticity forms a vortex that is strong enough to recover its "tail". This process can be followed in the successive triplet structures as they progress and evolve downstream. In an attempt to assess the effects of the fluctuations of the speed of the cylinder along its trajectory, we moved the cylinder along a new path in which the speed of motion relative to the fluid is kept constant. Although this motion nominally has the same values of frequency and amplitude as before, the cylinder really has acquired a slight streamwise oscillation relative to the steady translation of the earlier motion. Figure 1(b) shows the non-dimensional vorticity field for the constant-speed path. In this case, we have two single vortices shed in each cycle and the wake initially shows a relatively well-defined and close-packed Kármán arrangement that extends downstream for a distance of about 10 diameters. Later, however, a process of merging of neighboring vortices within either row takes place. This process forms a set of larger vortices with twice the downstream and cross-stream spacing of the original street and that we may call a "hyper-Kármán vortex-street".
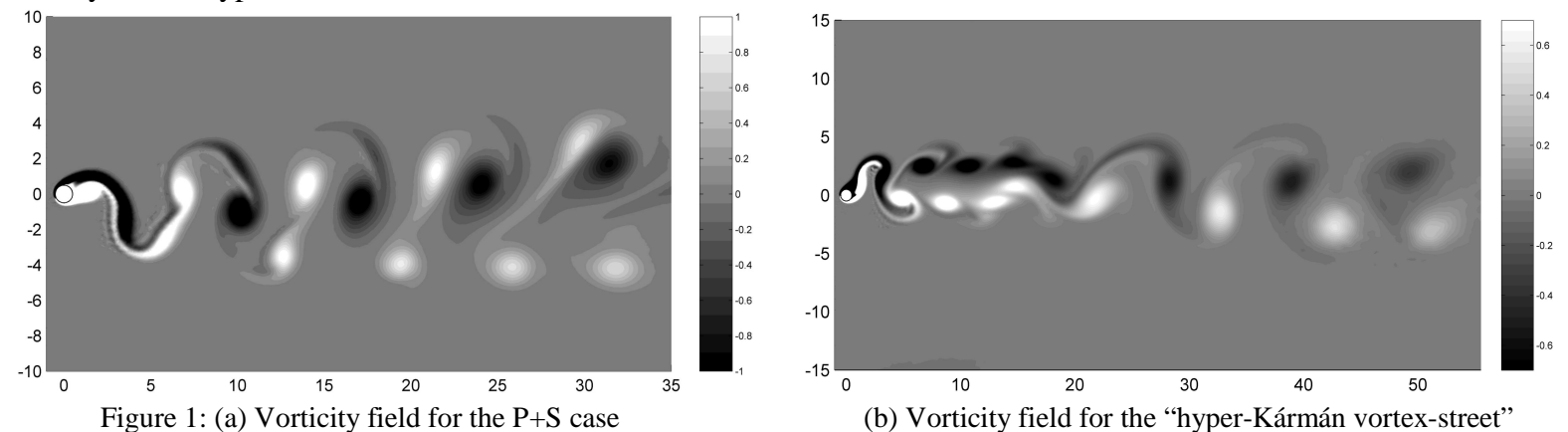

(b) Vorticity field for the "hyper-Kármán vortex-street"

\section{CONCLUSIONS}

We have introduced a mathematical-computational approach to solving flows in a frame of reference attached to a moving body and we have used this tool to explore the vortex synchronization regions in the amplitude-wavelength map of WR for low Reynolds number, focusing on the process of splitting which characterizes the formation of the $\mathrm{P}+\mathrm{S}$ and $2 \mathrm{P}$ structures. The formation of a secondary vortex street in the wake of a stationary cylinder in a uniform stream was observed by several researchers by using flow visualization techniques and hot-wire measurements on acoustically-forced cylinder wakes. In these experiments the primary Kármán vortex street decays downstream, and the secondary vortex street is formed with a larger scale of vortices (and thus a lower frequency) than those in the primary Kármán street. WR observed coalescence into a large-scale, Kármán-like arrangement of a large number of small vortices produced at high frequencies of the forced oscillation for sinusoidal trajectories. Working with constant-speed trajectories, we found the "hyper-Kármán vortex street" arrangement. This phenomenon, characterized by a pairwise merging process at low frequency that produces a duplication of the period, is, to the best of our knowledge, reported here for the first time for a moving, vertically oscillating cylinder. The synchronization of our primary vortex street with the vertical forced oscillation instead of with the Kármán shedding suggests that the pairwise merging phenomenon itself is related to the structure of the primary street (through the spacing of the vortices) rather than to the original process that generated the primary street. Based on point vortex models we are convinced that there exist a multitude of more complex vortex wake patterns (exotic wakes) that have not so far been seen in experiments. Although we understand how to "set these up" if we had complete control over how to distribute the vorticity in the flow, we do not yet fully understand how to produce such vortex wakes by oscillation and rotation of a wakeproducing bluff body. We hope to use the numerical tool developed here for such explorations in the future and plan to report our most recent findings in this regard at the Congress.

\section{References}

[1] Aref, H., Stremler, M. A.: On the motion of three point vortices in a periodic strip. J. Fluid Mech 314: 1-25, 1996.

[2] Khalak, A., Williamson, C. H. K.: Motion forces and mode transitions in vortex-induced vibration at low mass-damping. J. Fluids Struct. 13: 813851, 1999.

[3] Williamson, C. H. K., Roshko, A.: Vortex formation in the wake of an oscillating cylinder. J. Fluids Struct. 2: 355-381, 1988. 